emerging literature marking a change in how the digital footprints of 'modern migrants' might be conceptualised. 'Ora' incorporates AI driven meta data with real time immigration statistics and regional infectious disease prevalence, providing an early warning system for communicable diseases in transient populations.

The UN High Commission for Refugees now estimates that 70.8 million people are forcibly displaced around the world, the highest in recorded history with nearly one person displaced every two seconds. The growing social, humanitarian and economic costs signal a pressing need for collaborative innovation.

This multi-disciplinary approach highlights the benefits of cross-agency partnership in addressing the needs of a mobile and digitally connected global population. Agile development, prototyping and the clinical training of 'Ora' algorithms, were achieved through integration of workflows across clinicians, data scientists and technologists. Diversity in training, design approaches and backgrounds of the team yielded debate on the ethical and societal consequences of scraping meta data from vulnerable populations. Anecdotal evidence of European agencies using migrant smartphone data (social media, geolocation, messages) for deportation purposes led to the formation of 'Ora' operating values, and the emphasis of embedded bioethical principles in its deployment.

\section{AUDITED QUALITY IMPROVEMENT PROJECT ON OXYGEN PRESCRIBING ON AN ACUTE SURGICAL WARD}

Ali Shaladi, Stephen Crockett, Katharine Thomas. East and North Hertfordshire Trust, Lister Hospital, UK

\subsection{6/leader-2019-FMLM.53}

Oxygen is an important drug frequently used in the management of acutely unwell hospital patients. Studies have shown that deliberately increasing oxygen delivery in critically ill patients as well as high-risk surgical patients reduces organ failure, reduces length of ICU stay and, most importantly, improves mortality. British Thoracic Society Guidelines state that oxygen must be prescribed for all patients, with target saturations stipulated on the prescription for patient safety.

A quality improvement project was initiated and undertaken with the aim to improve the oxygen prescription rates across the surgical wards at Lister East and North Hertfordshire district general hospital over 2 months.

This project involved leading a team of surgical doctors to gather the relevant data on a weekly basis on compliance with oxygen prescribing looking at: COPD status, target saturations, timing, route and signature and dating of prescriptions. Teaching sessions were held based on the Results of our audit and targeted to the entire surgical department. The primary aim was to remind both junior and senior doctors and other allied healthcare prescribers alike the importance of oxygen prescribing and the lack of clear documentation on it. This presentation was accompanied by a systematic process of spearheading judicious interventions to appropriately promote the prescribing of oxygen.

On re-audit, the critical interventions stated led to an improvement in the documentation of target saturations $(13.6 \%$ to $42.2 \%)$, the route of administration $(15.9 \%$ to $28.9 \%)$, timing of oxygen delivery (11.4\% to $24.4 \%)$, and the signature and dating of the prescription (15.9\% to $40 \%)$.
An improvement in all aspects of oxygen prescribing was shown in our audit but with areas of enhancement still potentially achievable. By leading a team of doctors, an enrichment in the care of surgical of patients was achieved with regards to oxygen prescribing.

\section{$54 \quad$ BALINT GROUP AS THE TOOL FOR BUILDING HORIZONTAL STRUCTURE AND FOSTERING PATIENT- CENTRED CARE: REFLECTIONS ON RUNNING BALINT GROUP ON AN ORTHOPAEDIC WARD}

${ }^{1}$ Elena Nam, ${ }^{2}$ Paul Culatto. ${ }^{1}$ ST8, North West School of Psychiatry; ${ }^{2}$ ST6, North West School of Psychiatry

\subsection{6/leader-2019-FMLM.54}

The project took place on the emergency surgical and trauma unit at the Manchester Royal Infirmary. It was noted that patients with acute trauma would frequently include people with complex psychosocial difficulties.The intervention of Balint Group appeared to be most appropriate, which is unstructured reflective practice group initially created. It is important to note, that Balint group was selected both as intervention and assessment, as further reflections by the staff group would provide more insight into the nature of their difficulties.

We ran weekly 1-hour Balint Group session for 3 months. When running Balint Group a few main themes appeared, including 1) splitting up professional-patient relationship 2) avoidance of feeling 3) reduction of impact of responsibility by relying on superiors. Balint Group is effective in flattening hierarchy and helping the team to develop 'horizontal structure' with the free flow of ideas. In 'horizontal structure' professionals are accountable to patients and focussed on their care. Focus on professional-patient relationship and openness to difficult feelings is the building block of 'horizontal structure' in the team, which fosters growth and progress and high sensitivity to changing demands of environment, which are essential for effective leadership.

Balint group intervention in multidisciplinary team if a powerful assessment tool to explore the nature of the difficulties in the team, as well as it is a useful intervention, which helps to flatten hierarchy within the team, increase the flow of ideas from bottom up, and increase the sense of inidividual responsibility within the team. We present the qualitative and quantitative analysis, following the pilot project to support the benefit of running the Balint group in multidisciplinary team.

\section{DEVELOPING AND IMPLEMENTING A PEER TEACHING PROGRAMME FOR JUNIOR DOCTORS IN A TERTIARY TEACHING HOSPITAL}

Liam Loftus*, Claudia Maria Snudden. Royal Liverpool and Broadgreen University Hospitals Trust, UK

\subsection{6/leader-2019-FMLM.55}

It is widely accepted that a more engaged workforce Results in higher quality patient care. Education, particularly at junior doctor level, is a fantastic way to increase engagement.

All foundation doctors at The Royal Liverpool Hospital receive three hours of teaching every week from senior 